\title{
Engineering of lipid prodrug-based, hyaluronic acid-decorated nanostructured lipid carriers platform for 5-fluorouracil and cisplatin combination gastric cancer therapy
}

\author{
This article was published in the following Dove Press journal: \\ International Journal of Nanomedicine \\ 10 June 2015 \\ Number of times this article has been viewed
}

\section{Chun-Ying $\mathrm{Qu}^{1, *}$ \\ Min Zhoul,* \\ Ying-wei Chen ${ }^{2}$ \\ Mei-mei Chen ${ }^{3}$ \\ Feng Shen' \\ Lei-Ming $\mathrm{Xu}^{\prime}$}

'Digestive Endoscopic Diagnosis and Treatment Center, Xinhua Hospital, School of Medicine, Shanghai Jiaotong University, Shanghai, People's Republic of China; ${ }^{2}$ Shanghai Key Laboratory of Pediatric Gastroenterology and Nutrition, Shanghai, People's Republic of China; ${ }^{3}$ Digestive Department, Xinhua Hospital, School of medicine, Shanghai Jiaotong University, Shanghai, People's Republic of China

*These authors contributed equally to this work
Correspondence: Lei-Ming Xu Digestive Endoscopic Diagnosis and Treatment Center, Xinhua Hospital, School of Medicine, Shanghai Jiaotong University, Kongjiang Road 1665, Shanghai 200092, People's Republic of China

Email xuleimingsjtu@।63.com
Purpose: The first-line chemotherapy treatment protocol for gastric cancer is combination chemotherapy of 5-fluorouracil (5-FU) and cisplatin (CDDP). The aim of this study was to engineer prodrug-based nanostructured lipid carriers (NLC) platform for codelivery of 5-FU and CDDP to enhance therapy and decrease toxicity.

Methods: First, 5-FU-stearic acid lipid conjugate was synthesized by two steps. Second, 5-FU-stearic acid prodrug and CDDP were loaded in NLC. Finally, hyaluronic acid (HA) was coated onto NLC surface. Average size, zeta potential, and drug loading capacity of NLC were evaluated. Human gastric cancer cell line BGC823 (BGC823 cells) was used for the testing of in vitro cytotoxicity assays. In vivo antitumor activity of NLC was evaluated in mice bearing BGC823 cells model.

Results: HA-coated 5-FU-stearic acid prodrug and CDDP-loaded NLC (HA-FU/C-NLC) showed a synergistic effect in combination therapy and displayed the greatest antitumor activity than all of the free drugs or uncoated NLC in vitro and in vivo.

Conclusion: This work reveals that HA-coated NLC could be used as a novel carrier to codeliver 5-FU and CDDP for gastric cancer therapy. HA-FU/C-NLC could be a promising targeted and combinational therapy in nanomedicine.

Keywords: gastric cancer, nanostructured lipid carriers, hyaluronic acid, combination chemotherapy, lipid prodrug

\section{Introduction}

Gastric cancer (GC) is the fourth most common cancer worldwide and second leading cause of cancer mortality. ${ }^{1}$ According to tumor characteristics and stage, current treatment scheme comprises surgery, chemotherapy, radiation therapy, or combination of these two or three treatments. Due to the lack of clearly defined symptoms during the early stage of GC, the patients are usually diagnosed in the advanced or metastatic stage, and prognosis for GC remains poor, with a 5-year survival rate of only $20 \%-25 \%$ when surgery is performed alone..$^{2-4}$ According to National Comprehensive Cancer Network (NCCN) guidelines for GC in 2012, chemotherapy is the mainstay of palliative therapy for advanced or metastatic disease. However, even two- or three-drug regimens still have remarkable toxicity which is often unacceptable in elderly patients or those with comorbidities. ${ }^{2}$ Therefore, it is necessary to develop new agents or novel drug delivery systems to confer survival benefits with acceptable tolerability.

Nanostructured lipid carriers (NLC), a new generation of solid lipid nanoparticles (SLN), consist of solid lipid matrix with certain amount of liquid lipid. Because of their 
less ordered inner structure, most of the lipids are included in the category of GRAS and have low toxicity. NLC drug delivery systems possess several advantages in cancer chemotherapy, such as higher drug loading capacity and stability during storage, good biocompatibility, controllable drug release, and feasibility of scaling up. ${ }^{5-7}$ However, the inherent structure of NLC contributes to the hydrophobic nature of these lipids, which limits their use in the encapsulation of hydrophilic drugs. Lipophilic modification is one of the most effective methods for the encapsulation of these drugs. ${ }^{8}$ In this paper, stearic acid was chosen as both the solid lipid of NLC and the lipophilic group, and was conjugated with 5-fluorouracil (5-FU).

The effectiveness of NLC could be further improved by targeting tumors with ligands coated to the surface of NLC. Hyaluronic acid (HA) is a biodegradable, polyanionic glycoaminoglycan with repetitive disaccharide units of D-glucuronic acid and D- $N$-acetylglucosamine. ${ }^{9}$ It has been identified as a potent targeting ligand for detecting tumors possessing CD44 over-expressing cells. ${ }^{6,10,11}$ Several studies have identified CD44 as a cell surface marker of GC stem cells. Recent research has reported that CD44 expression in GC could apply as a prognostic indicator for tumor progression, metastasis, and patient survival. ${ }^{12,13}$ Moreover, HA has been identified to provide a potential strategy to selectively target and efficiently eradicate GC. ${ }^{14,15}$ Therefore, HA was selected as a targeting ligand for $\mathrm{GC}$ in this paper.

Cisplatin (CDDP) plus FU are reasonable first-line options and globally accepted first-line regimens for GC chemotherapy ${ }^{16-18}$ The meta-analysis by Cervantes et al has demonstrated a significant benefit of survival for the combination regimens (5-FU and CDDP). ${ }^{19}$ However, toxicity was increased in the combination schedules, and combination therapy should be considered only in patients with good performance status. ${ }^{19}$ To reduce the toxicity and improve therapeutic efficacy, 5-FU prodrug was synthesized and 5-FU prodrug and CDDP-loaded NLC were designed.

5 -FU is a fluoropyrimidine antimetabolite and widely used in the treatment of GC. The clinical use of 5-FU is constantly challenged by its poor selectivity and various side effects such as myelosuppression, mucositis, nausea, emesis, and hand-foot syndrome. ${ }^{20,21}$ The 5-FU regimen has poor biopharmaceutical characteristics, such as poor absorption, rapid drug catabolism, and short biological halflife (10-20 minutes), that hinder its clinical application. ${ }^{22,23}$ Several strategies to minimize these side effects have been researched. They can be divided into two kinds of methods. One kind is chemical modification, such as synthesis of low molecular weight 5-FU derivatives, ${ }^{24}$ conjugation with polymeric materials, ${ }^{25}$ conjugation with peptide, ${ }^{26}$ or modification with palmitic acid. ${ }^{22}$ The other kind is based on nanocarriers, such as liposomes, ${ }^{27}$ nanoparticles, ${ }^{28}$ micelles, ${ }^{29,30}$ and SLN. ${ }^{31}$ Combining the advantages of these two strategies, we designed a novel platform: lipid prodrug NLC to codeliver 5-FU and CDDP. Compared to the aforementioned chemical modification, the use of lipid prodrugs in nanocarriers provides important benefits: facile cleavage of the ester bond, sustained drug release, higher amount of prodrug loading, and reduced adverse effects. ${ }^{32}$ Additionally, structural similarities between NLC and lipid prodrugs may facilitate prodrug loading/insertion. ${ }^{8}$

CDDP is extensively used for the treatment of various cancers such as ovarian, gastric, colorectal, and other severe carcinomas. In clinical use, maximum tolerated dose of CDDP is significantly limited due to nephrotoxicity ${ }^{33} \mathrm{In}$ order to reduce the toxicity, nanoparticles and liposomes have been used. ${ }^{34-36}$ CDDP is poorly soluble in water and can be loaded into NLC.

In this paper, 5-FU-stearic acid conjugate (prodrug) was synthesized by conjugation of the ester bond with stearic acid. Then, 5-FU-stearic acid and CDDP were encapsulated into NLC by emulsification and low-temperature solidification method. Finally, HA decorated 5-FU prodrug and CDDP NLC were prepared. The in vitro and in vivo antitumor efficacy was assessed in human GC cell line BGC 823 . This system was anticipated to achieve stable, controlled release and active targeting lipid prodrug NLC of 5-FU and CDDP, to improve synergistic anticancer effects and reduce toxicity.

\section{Materials and methods Materials}

5-FU was provided by Shandong Qilu Pharmaceutical Co., Ltd. (Jinan, People's Republic of China). CDDP was provided by Nanjing Pharmaceutical Factory Co., Ltd. (Nanjing, People's Republic of China). Glyceryl monostearate was purchased from Shanghai Chineway Pharmaceutical Tech. Co., Ltd. (Shanghai, People's Republic of China). Injectable soybean oil was purchased from Guangzhou Hanfang Pharmaceutical Co., Ltd. (Guangzhou, People's Republic of China). Injectable soya lecithin was obtained from Shanghai Taiwei Pharmaceutical Co., Ltd (Shanghai, People's Republic of China). HA was provided by Shandong Freda Biochem Co., Ltd. Stearic acid, Tween ${ }^{\circledR}$ 80, dimethyldioctadecylammonium bromide, and Roswell Park Memorial Institute 1640 medium were purchased from Sigma-Aldrich Co., Ltd (St Louis, MO, USA). All other chemicals were of analytical or higher grade. 


\section{Cell line and cell culture}

Human GC cell line BGC823 was obtained from the China Center for Type Culture Collection (CCTCC) by CrownBio, Beijing, China. BGC823 cell line was cultured in Roswell Park Memorial Institute 1640 medium supplemented with $10 \%$ heat-inactivated fetal bovine serum under humidified conditions at $37^{\circ} \mathrm{C}$ and $5 \% \mathrm{CO}_{2}$.

\section{Animals}

Four-week-old BALB/c nude mice ( $20 \mathrm{~g}$ body weight) were purchased from the Shanghai SLAC laboratory Animal Co., Ltd. All animal experiments complied with the Animal Management Rules for the administration of experimental animals (Order No 54 of the Shanghai Municipal Government).

\section{Synthesis of 5-FU-stearic acid}

The synthetic route of 5-FU-stearic acid is shown in Figure 1. First, $N, N^{\prime}$-1,3-bis(hydroxymethyl-5-fluorouracil) was synthesized. In brief, 5-FU (10 mmol), formaldehyde ( $25 \mathrm{mmol})$, and water $(10 \mathrm{~mL})$ were added to a round bottom flask. Then, the mixed solution was immersed in an oil bath at $60^{\circ} \mathrm{C}$ and reacted for 6 hours under agitation..$^{22,37}$ Finally, $N, N^{\prime}$-1,3-bis(hydroxymethyl-5-fluorouracil) was obtained via rotary evaporation method and purification by column chromatography.

Then, 5-FU-stearic acid was obtained by the facile esterification reaction. ${ }^{38}$ Stearic acid $(10 \mathrm{mmol})$, $N, N^{\prime}$-dicyclohexylcarbodiimide (20 mmol), and $N, N^{\prime}-1$, 3-bis(hydroxymethyl-5-fluorouracil) $(5 \mathrm{mmol})$ in dichloromethane with the catalyst 4-dimethylaminopyridine were stirred at room temperature for 48 hours. $N, N^{\prime}$-Dicyclohexylurea was removed by filtration. Finally, 5-FU-stearic acid was obtained by precipitation with diethyl ether and purified by washing with acetone for three times. ${ }^{1} \mathrm{H}$ NMR spectra of 5-FU-stearic acid was analyzed on an NMR spectrometer (300 Hz, Bruker, Switzerland).

\section{Preparation of 5-FU-stearic acid prodrug and CDDP-loaded NLC (FU/C-NLC)}

5-Flourouracil-stearic acid prodrug and CDDP-loaded NLC (FU/C-NLC) were prepared by emulsification and low-temperature solidification method. ${ }^{39,40}$ Briefly, the lipid phase, comprising glyceryl monostearate, soya lecithin, soybean oil (2:1:1,w/w/w), 5-FU-stearic acid, and CDDP, was obtained by melting and dissolving at $70^{\circ} \mathrm{C}$. The lipid phase was rapidly injected into the aqueous phase $(10 \mathrm{~mL})$ containing Tween-80 (100 mg) and dimethyldioctadecylammonium bromide $(30 \mathrm{mg}$ ) at the same temperature under stirrer $(800 \mathrm{rpm})$ at $30^{\circ} \mathrm{C}$ for 3 hours. Then, the nano-emulsion was immediately transferred to a flask containing $20 \mathrm{~mL}$ distilled water in an ice bath $\left(0^{\circ} \mathrm{C}-2^{\circ} \mathrm{C}\right)$. Finally, FU/C-NLC were harvested by stirring in an ice bath for an additional 1 hour.

FU-NLC, C-NLC, and blank NLC were prepared by the same method mentioned earlier without adding CDDP, 5-FU-stearic acid, or both drugs, respectively.<smiles></smiles>

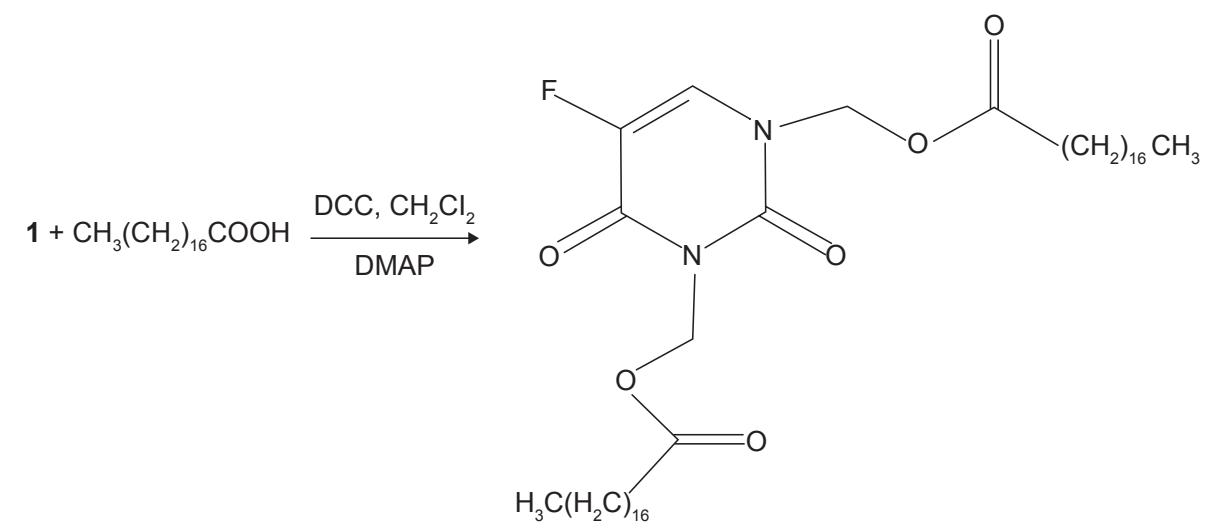

Figure I Synthetic route of 5-FU-stearic acid.

Abbreviations: FU, fluorouracil; DCC, N,N'-dicyclohexylcarbodiimide; DMAP, 4-dimethylaminopyridine. 


\section{Preparation of HA-coated FU/C-NLC (HA-FU/C-NLC)}

HA-coated FU/C-NLC (HA-FU/C-NLC) were prepared by electrostatic attraction. ${ }^{6}$ In brief, $1 \mathrm{~mL}$ of FU/C-NLC dispersion was added drop wise into $0.05 \%, 0.1 \%, 0.2 \%, 0.3 \%$, and $0.4 \%$ of HA solutions (w/v) that were stirred at $600 \mathrm{rpm}$ at room temperature, and then stirred for 2 hours to complete the modification. Subsequently, free HA was removed from HA-FU/C-NLC by gel chromatography. NLC were purified and obtained by filtering through a $0.45 \mu \mathrm{m}$ membrane.

\section{Characterization of NLC}

The surface morphologies of NPs were examined by transmission electronic microscopy. The particle size, polydispersity index, and zeta potential of HA-FU/C-NLC, FU/C-NLC, FU-NLC, and C-NLC were analyzed using photon correlation spectroscopy with a Zetasizer 3000 (Malvern Instruments, Malvern, England). The average particle size was expressed as volume mean diameter.

Encapsulation efficiency (EE) of 5-FU-stearic acid or CDDP in NLC was assessed by the indirect method. In brief, the NLC dispersion was precipitated by the $\mathrm{pH}$ adjustment. After centrifugation, the drug content in the supernatant was determined.

The content of 5-FU-stearic acid was estimated by high performance liquid chromatography (HPLC) (Waters 2695, Milford, MA, USA). ${ }^{31}$ Chromatographic separations were carried out using the Intertsil ${ }^{\circledR}$ ODS-3V $(250 \mathrm{~mm} \times 4.6 \mathrm{~mm}$, $5 \mu \mathrm{m})$. Mobile phase consisted of a mixture of methanol and water $(30: 70, \mathrm{v} / \mathrm{v})$. Flow rate was kept at $1.0 \mathrm{~mL} / \mathrm{min}$ and the system was maintained at $30^{\circ} \mathrm{C}$, and the detection was carried out at $\lambda=266 \mathrm{~nm}$. Injection volume was $20 \mu \mathrm{L}$.

The content of CDDP was determined by graphite furnace atomic absorption spectroscopy. The detection was carried out at $\lambda=265.9 \mathrm{~nm}$ and slit bandwidth of $0.70 \mathrm{~nm}^{41}$

EE of NLC was calculated from the following equation:

$$
\mathrm{EE}(\%)=\left(\mathrm{W}_{\text {total }}-\mathrm{W}_{\text {free }}\right) / \mathrm{W}_{\text {total }} \times 100
$$

where $\mathrm{W}_{\text {total }}$ is the weight of 5-FU-stearic acid or CDDP added when preparing NLC and $\mathrm{W}_{\text {free }}$ is the weight of the 5-FU-stearic acid or CDDP determined in the supernatant of NLC.

\section{In vitro drug release profiles}

In vitro release studies of different NLC formulations were assessed in release medium $(0.1 \%$ Tween 80 in phosphate buffered saline, $\mathrm{pH}$ 7.4) by the dialysis method. NLC samples
$(5 \mathrm{~mL})$ were enclosed in dialysis bags, and incubated with $50 \mathrm{~mL}$ of release medium at $37^{\circ} \mathrm{C}$ under mild agitation in a water bath. At predetermined time points, samples $(1 \mathrm{~mL})$ were withdrawn from the incubation medium and analyzed for 5-FU-stearic acid by the HPLC method or for CDDP by the GFAAS method. After sampling, the release medium was replaced by fresh phosphate buffered saline.

\section{Cell toxicity assays}

Cell toxicities of different formulations were evaluated in BGC823 cells with MTT assay. Cells were seeded in 96-well culture plates at a density of $1 \times 10^{4}$ cells per well and incubated for 24 hours. Then, cells were reseeded with blank NLC, HA-FU/C-NLC, FU/C-NLC, FU-NLC, C-NLC, 5-FU-stearic acid + CDDP solution (5-FU:CDDP $=40: 1,30: 1$, 20:1, 10:1, 5:1; w/w), 5-FU-stearic acid solution, and CDDP solution at various concentrations for 48 hours at $37^{\circ} \mathrm{C}$ and $5 \% \mathrm{CO}_{2}$ atmosphere. Culture medium was used as the blank. Then, MTT solution $(5 \mathrm{mg} / \mathrm{mL})$ was added to each well and cells were incubated for another 4 hours. After removing the unreduced MTT and medium, each well was replaced with $200 \mu \mathrm{L}$ of dimethylsulfoxide (DMSO) to dissolve the MTT formazan crystals. The absorbance (A) of formazan product was measured using a microplate reader (Model 680, Bio-Rad, Hercules, CA, USA) at $570 \mathrm{~nm}$. The relative cell viability $(\mathrm{CV})$ was calculated from the following equation:

$$
\mathrm{CV}(\%)=\left(\mathrm{A}_{\text {sample }}-\mathrm{A}_{\text {blank }}\right) /\left(\mathrm{A}_{\text {control }}-\mathrm{A}_{\text {blank }}\right) \times 100
$$

Half maximal inhibitory concentration $\left(\mathrm{IC}_{50}\right)$ values of NLC were calculated. Combination index (CI) was determined to study the synergy between 5-FU-stearic acid and CDDP in the combination system. ${ }^{42} \mathrm{CI}$ was calculated from the following equation:

$$
\mathrm{CI}_{50}=(\mathrm{D})_{1} /\left(\mathrm{D}_{50}\right)_{1}+(\mathrm{D})_{2} /\left(\mathrm{D}_{50}\right)_{2}
$$

where $\mathrm{CI}_{50}$ represents the combination index calculated when using the $\mathrm{IC}_{50}$ values of the drugs, (D) and (D) $)_{2}$ represent the concentrations of 5-FU-stearic acid and CDDP, respectively, in the combination system at the $\mathrm{IC}_{50}$ value; $\left(\mathrm{D}_{50}\right)_{1}$ and $\left(\mathrm{D}_{50}\right)_{2}$ represent the $\mathrm{IC}_{50}$ values of 5-FU-stearic acid alone and CDDP alone, respectively. $\mathrm{CI}_{50}<1$ represents synergism, and $\mathrm{CI}_{50}>1$ represents antagonism.

\section{In vivo anticancer efficacy}

$\mathrm{BALB} / \mathrm{c}$ nude mice bearing BGC823 gastric tumor $\left(\sim 90-100 \mathrm{~mm}^{3}\right)$ were randomly divided into eight groups $(\mathrm{n}=6)$, and $0.9 \%$ saline, HA-FU/C-NLC, FU/C-NLC, FU-NLC, C-NLC, 5-FU-stearic acid + CPPD solution, 
5-FU-stearic acid solution, and CDDP solution were injected, respectively. The amounts of drugs used for each group in the formulations mentioned earlier were $20 \mathrm{mg} 5-\mathrm{FU} / \mathrm{kg}$ and $1 \mathrm{mg} \mathrm{CDDP} / \mathrm{kg}$. Mice of each group were given the formulations by tail vein injection once every week. Twenty-one days later, all the mice were sacrificed by cervical dislocation and the tumor tissues were taken out.

Tumor volume $\left(\mathrm{mm}^{3}\right)$ of each mouse was measured with a digital caliper every 3 days, and was calculated according to the following equation:

$$
\text { Tumor volume }\left(\mathrm{mm}^{3}\right)=\mathrm{L} \times \mathrm{W}^{2} / 2
$$

where $\mathrm{L}$ and $\mathrm{W}$ represent the largest diameter and the smallest diameter, respectively. The weight of each mouse was measured every 3 days after injection.

\section{Statistical analysis}

All experiments were performed at least three times $(n=3)$, and expressed as mean \pm SD (standard deviation). Statistical differences were determined using two-tailed Student's $t$-test or one-way analysis of variance. The significance level was taken as $95 \%(P<0.05)$.

\section{Results and discussion Structural characterization of 5-FU- stearic acid}

The chemical structure of 5-FU-stearic acid was confirmed using ${ }^{1} \mathrm{H}$ NMR spectroscopy. ${ }^{1} \mathrm{H}$ NMR (DMSO-d, $300 \mathrm{mHz}$ ): $\delta 7.39$ $(\mathrm{t},=\mathrm{C}-\mathrm{N}-,=\mathrm{C}-\mathrm{F}-), 5.95(\mathrm{~d},-\mathrm{CO}-\mathrm{O}-\mathrm{C}-,-\mathrm{CO}-\mathrm{N}-\mathrm{C}-), 2.25$ $(\mathrm{s},-\mathrm{O}-\mathrm{CO}-\mathrm{C}-), 1.68-1.29\left(\mathrm{q},-\mathrm{CH}_{2}-\right), 0.96\left(\mathrm{t},-\mathrm{CH}_{3}\right)$. The structures of $-\mathrm{CO}-\mathrm{O}-\mathrm{C}-,-\mathrm{CO}-\mathrm{N}-\mathrm{C}-$, and $-\mathrm{O}-\mathrm{CO}-\mathrm{C}-$ confirmed the formation of 5-FU-tearic acid conjugate. The yield was 78.9\%.

5-Flourouracil is a major drug used in the treatment of GC. ${ }^{43}$ The clinical use of 5-FU is normally accompanied by various side effects. ${ }^{22}$ The treatment by 5 -FU has several shortcomings, such as rapid drug catabolism, poor absorption, short biological half-life of the drug, and non-selective action against healthy cells of gastrointestinal tract and bone marrow. ${ }^{44}$ Synthesis of 5-FU derivatives with low molecular weight, conjugation with other compounds, and encapsulation in nanocarriers can minimize these side effects. ${ }^{45}$ The encapsulation of 5-FU in hydrophobic materials will be made feasible by a lipid-based prodrug approach. ${ }^{46}$ The metabolic pathways of fatty acids, facile cleavage of the ester bond of lipid-drug conjugate, and a higher amount of prodrug loading inside the nanoparticles are some of the potential benefits of this type of approach. In this research, we introduced NLC as outstanding lipid carriers and used HA coating on the prodrug-loaded NLC to improve their targeted antitumor efficiency.

\section{Determination of HA concentration coating on NLC surface}

HA can selectively target GC cells. ${ }^{15}$ HA could specifically recognize its receptors, CD44, and has been identified as a potent targeting ligand for tumors possessing CD44 overexpressing GC cells. ${ }^{47}$ Furthermore, the HA coating provides a hydrophilic shield, similar to polyethylene glycol, for promotion of long blood circulation. ${ }^{48} \mathrm{HA}$ was also selected for its favorable properties such as good biocompatibility, high biodegradability, low toxicity, and non-immunotoxicity. ${ }^{49}$ To select the suitable concentration for the sufficient coating of NLC, $0.05 \%, 0.1 \%, 0.2 \%, 0.3 \%$, and $0.4 \%$ concentrations of HA solution (w/v) were prepared and used for the formation of HA-FU/C-NLC. HA is a polyanion electrolyte molecule with a large number of negative charges. As the amount of HA increases on cationic NLC surface, zeta potential of the particles decreases. No obvious decrease of potential may indicate the completion of coating. Furthermore, particle size of NLC would enlarge on coating with HA ligands. However, excessive coating of ligands may cause the aggregation of the particles, leading to a sudden increase in particle size..$^{50}$ Additionally, the modification by HA should not influence the EE of the FU/C-NLC. The optimum formulation was determined by measuring the zeta potential, particle size, and EE. As shown in Table 1, zeta potential decreased from $0.05 \%$ to $0.2 \%$ of HA concentration, and after that the potential was stable. The size was suddenly increased from $180 \mathrm{~nm}$ to over

Table I Determination of concentration of HA coating on NLC surface

\begin{tabular}{|c|c|c|c|c|}
\hline $\begin{array}{l}\text { HA } \\
\text { concentration }\end{array}$ & $\begin{array}{l}\text { Zeta } \\
\text { potential }(\mathbf{m V})^{*}\end{array}$ & $\begin{array}{l}\text { Particle } \\
\text { size }(\mathbf{n m})^{*}\end{array}$ & $\begin{array}{l}\text { Encapsulation } \\
\text { efficiency of } 5 \text {-FU (\%)* }\end{array}$ & $\begin{array}{l}\text { Encapsulation efficiency } \\
\text { of CDDP (\%)* }\end{array}$ \\
\hline $0.05 \%$ & $+37.5 \pm 3.1$ & $99.5 \pm 2.1$ & $92.5 \pm 2.5$ & $89.9 \pm 3.1$ \\
\hline $0.1 \%$ & $+33.9 \pm 2.9$ & $128.3 \pm 3.7$ & $91.6 \pm 2.1$ & $88.6 \pm 3.7$ \\
\hline $0.2 \%$ & $+26.4 \pm 2.3$ & $181.2 \pm 3.1$ & $90.5 \pm 2.6$ & $89.5 \pm 2.9$ \\
\hline $0.3 \%$ & $+25.9 \pm 2.6$ & $185.7 \pm 9.3$ & $78.4 \pm 3.5$ & $61.6 \pm 7.1$ \\
\hline $0.4 \%$ & $+26.1 \pm 2.2$ & $348.9 \pm 25.6$ & $69.1 \pm 3.9$ & $49.1 \pm 6.1$ \\
\hline
\end{tabular}

Note: *Data is presented as mean \pm standard deviation.

Abbreviations: HA, hyaluronic acid; NLC, nanostructured lipid carriers; FU, fluorouracil; CDDP, cisplatin. 


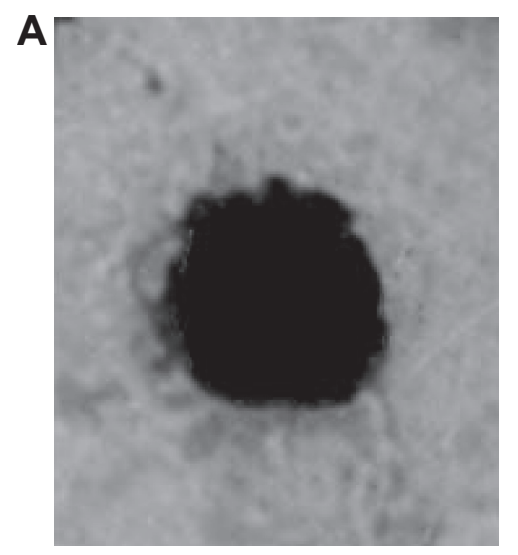

FU/C-NLC

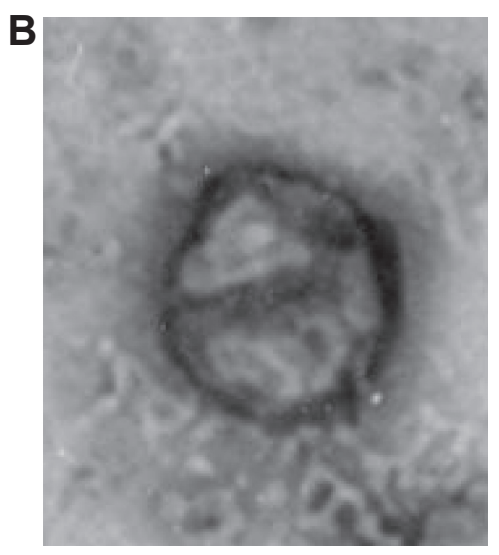

HA-FU/C-NLC

Figure 2 TEM imaging of (A) FU/C-NLC and (B) HA-FU/C-NLC.

Abbreviations: TEM, transmission electronic microscopy; HA, hyaluronic acid; FU, fluorouracil; CDDP, cisplatin; C, CDDP-loaded; NLC, nanostructured lipid carriers.

$340 \mathrm{~nm}$ at the concentration of $0.4 \%$, which demonstrates that this concentration is not suitable for the coating process. The EEs of 5-FU and CDDP were approximately $90 \%$ at the HA concentration between $0.05 \%$ and $0.2 \%$, while at the higher concentrations, EE was significantly decreased. So, a concentration of $0.2 \%$ was determined to be the ideal concentration of HA and was used for the coating experiments.

\section{Characterization of NLC}

The transmission electron micrograph pictures of the HAFU/C-NLC and FU/C-NLC are shown in Figure 2. HA-FU/CNLC has gray coats on the black spherical shaped particles, which were different in appearance with the black FU/C-NLC spherical particles.

The particle size, zeta potential, and EE of different NLC complexes are shown in Table 2. The size of HA-FU/C-NLC was $182 \mathrm{~nm}$, with a zeta potential of $+26 \mathrm{mV}$. Positive charge of the carriers could exploit the negative charge present at the cell surface, promote the cell penetration, and thus facilitate the internalization process of the particles. ${ }^{51}$ The EEs of 5-FU and CDDP loaded in HA-FU/C-NLC were $90 \%$ and $89 \%$, respectively. EEs have marked influence on drug therapeutic effect. High EE could offer advantages in the in vitro and in vivo studies.

In vitro 5-FU release profiles of HA-FU/C-NLC, FU/CNLC, and FU-NLC are illustrated in Figure 3. Over $80 \%$ of drug release was obtained by HA-FU/C-NLC, FU/C-NLC, and FU-NLC at 48, 36, and 24 hours, respectively. HAFU/C-NLC was the most sustained-release formulation. This may be due to the reason that the coating of HA ligands controlled the 5-FU-stearic acid prodrug release. The same phenomenon was found in the CDDP release profiles of HAFU/C-NLC, FU/C-NLC, and C-NLC (Figure 4). HA-FU/CNLC showed $80 \%$ accumulated CDDP release at 48 hours, while the release of FU/C-NLC and C-NLC completed at 12 hours of study. The sustained-release behavior may help with the in vivo antitumor effect, which can be observed in the following experiments.

\section{Cell toxicities in BGC823 cells}

Evaluation of drug-drug interaction is important in all areas of medicine, and particularly in cancer chemotherapy where combination therapy is commonly used. ${ }^{52} \mathrm{CI}$ analyses are the most popular methods for evaluating drug interactions in

Table 2 Physicochemical characterization of different NLC complexes

\begin{tabular}{llllll}
\hline Formulation & Particle size $(\mathbf{n m})$ & PDI & Zeta potential $(\mathbf{m V})$ & EE of 5-FU (\%) & EE of CDDP $(\%)$ \\
\hline Blank NLC & $95.8 \pm 2.1$ & $0.12 \pm 0.01$ & $+45.3 \pm 3.5$ & NA & NA \\
FU-NLC & $131.4 \pm 2.3$ & $0.16 \pm 0.02$ & $+37.3 \pm 2.3$ & $91.9 \pm 1.9$ & NA \\
C-NLC & $118.2 \pm 2.8$ & $0.13 \pm 0.02$ & $+35.1 \pm 1.9$ & NA & $89.5 \pm 2.8$ \\
FU/C-NLC & $145.3 \pm 2.9$ & $0.19 \pm 0.03$ & $+29.7 \pm 2.1$ & $91.1 \pm 2.5$ & $88.7 \pm 3.1$ \\
HA-FU/C-NLC & $181.6 \pm 3.2$ & $0.21 \pm 0.04$ & $+26.3 \pm 2.4$ & $89.8 \pm 2.9$ & $89.1 \pm 2.1$ \\
\hline
\end{tabular}

Note: Data is presented as mean \pm standard deviation.

Abbreviations: NLC, nanostructured lipid carriers; PDI, polydispersity index; EE, encapsulation efficiency; FU, fluorouracil; CDDP, cisplatin; NA, not applicable; C, CDDPloaded; HA, hyaluronic acid. 


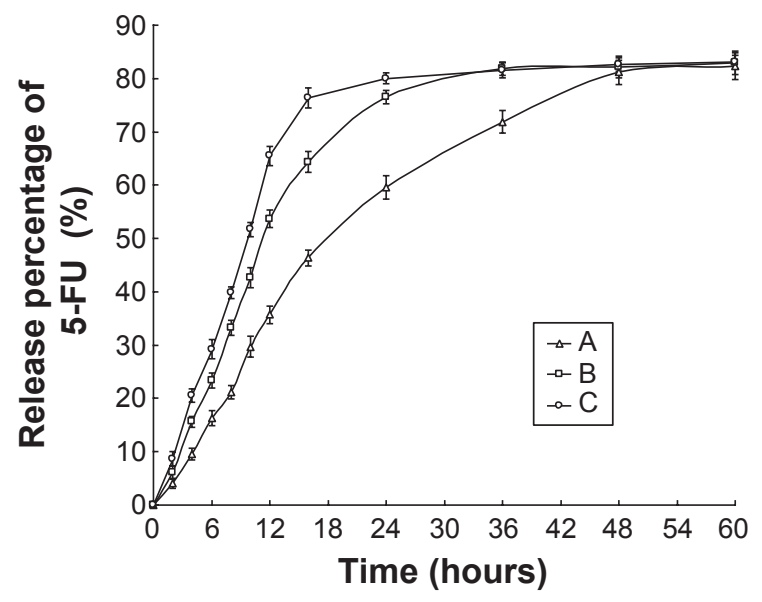

Figure 3 In vitro 5-FU release profiles of HA-FU/C-NLC (A), FU/C-NLC (B), and FU-NLC (C).

Abbreviations: 5-FU, fluorouracil; HA, hyaluronic acid; FU, fluorouracil; CDDP, cisplatin; C, CDDP-loaded; NLC, nanostructured lipid carriers.

combination cancer chemotherapy. The interaction between 5-FU and CDDP can be investigated by CI, where $\mathrm{CI}<1$, $\mathrm{CI}=1$, and $\mathrm{CI}>1$ indicate synergism, additive, and antagonism, respectively. ${ }^{53}$ In this study, $\mathrm{CI}_{50}$ values were first calculated in free drug samples and then further in drug-loaded NLC formulations.

Free 5-FU-stearic acid and free CDDP combinations at various 5-FU to CDDP weight ratios (40:1, 30:1, 20:1, 10:1, $5: 1, \mathrm{w} / \mathrm{w}$ ) were applied in BGC823 cells with MTT assay to determine the effective combination proportion of these two free drugs. As shown in Table 3, the combination therapy showed synergistic effect when 5-FU to CDDP weight ratios were 20:1 $\left(\mathrm{CI}_{50}=0.81\right)$ and 10:1 $\left(\mathrm{CI}_{50}=0.98\right)$. Next, 5-FU-stearic acid and CDDP containing NLC (FU/C-NLC and HA-FU/C-NLC) at the 5-FU to CDDP ratios of 20:1 and 10:1 were prepared and analyzed (Table 4). The weight ratio of 20:1 exhibited stronger synergism in both coated and uncoated NLC formulations, suggesting the best 5-FU to CDDP ratios in the nanocarrier formulas. This ratio was used for the in vivo antitumor study.

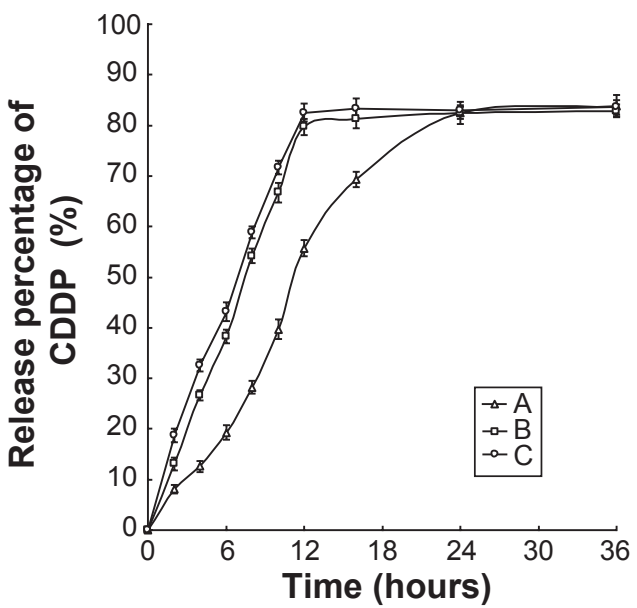

Figure 4 In vitro CDDP release profile of HA-FU/C-NLC (A), FU/C-NLC (B), and C-NLC (C).

Abbreviations: CDDP, cisplatin; C, CDDP-loaded; HA, hyaluronic acid; FU, fluorouracil; NLC, nanostructured lipid carriers.

\section{In vivo anticancer efficacy}

According to the cell toxicity results mentioned earlier, the in vivo anticancer efficacy and systemic toxicity of different formulations were further investigated on mice bearing BGC823 human GC xenografts. The tumor growth inhibitions and body weight changes in different groups were monitored for 21 days. Figure 5 clearly demonstrates that tumor growth was significantly inhibited by NLC formulations than drug solution groups $(P<0.05)$. The combination of 5-FU and CDDP formulas were more effective than their single drug counterparts in both free drugs and NLC groups $(P<0.05)$. The HA-FU/C-NLC showed the greatest antitumor activity than all of the free drugs or uncoated NLC formulations $(P<0.05)$. Systemic toxicity is a severe side effect during the cancer treatment. In this study, NLC formulations and surface coating by targeted ligands were designed to overcome this problem. Changes in the body weight of tumor-bearing mice after injections can indicate the systemic toxicity. The body weights of FU/C-NLC, FU-NLC, and C-NLC groups increased slightly, which

Table $3 \mathrm{IC}_{50}$ and $\mathrm{Cl}_{50}$ values of 5-FU-stearic acid and CDDP solution with various 5-FU/CDDP ratios

\begin{tabular}{|c|c|c|c|c|}
\hline Drug solutions & 5-FU/CDDP ratio (w/w) & $\mathrm{IC}_{50} 5$-FU $(\mu \mathrm{g} / \mathrm{mL})$ & $I C_{50}$ CDDP $(\mu \mathrm{g} / \mathrm{mL})$ & $\mathrm{Cl}_{50}$ \\
\hline 5-FU-stearic acid & NA & 37.52 & NA & NA \\
\hline CDDP & NA & NA & 3.39 & NA \\
\hline 5-FU-stearic acid + CDDP & $40: 1$ & 35.2 & 0.88 & 1.20 \\
\hline 5-FU-stearic acid + CDDP & 30:1 & 30.7 & 1.02 & 1.12 \\
\hline 5-FU-stearic acid + CDDP & $20: 1$ & 19.5 & 0.98 & 0.81 \\
\hline 5-FU-stearic acid + CDDP & $10: 1$ & 17.5 & 1.75 & 0.98 \\
\hline 5-FU-stearic acid + CDDP & $5: 1$ & 14.6 & 2.92 & 1.25 \\
\hline
\end{tabular}

Abbreviations: $\mathrm{IC}_{50}$, half maximal inhibitory concentration; $\mathrm{Cl}_{50}$, combination index calculated when using the $\mathrm{IC}_{50}$ values of the drugs; FU, fluorouracil; $C D D P$, cisplatin; NA, not applicable. 
Table $4 \mathrm{IC}_{50}$ and $\mathrm{Cl}_{50}$ values of NLC formulations with different 5-FU/CDDP ratios

\begin{tabular}{lllll}
\hline $\begin{array}{l}\text { Drug } \\
\text { solutions }\end{array}$ & $\begin{array}{l}\text { 5-FU/CDDP } \\
\text { ratio }(\mathbf{w} / \mathbf{w})\end{array}$ & $\begin{array}{l}\mathrm{IC}_{50} \text { 5-FU } \\
(\mu \mathrm{g} / \mathrm{mL})\end{array}$ & $\begin{array}{l}\mathrm{IC}_{50} \mathrm{CDDP} \\
(\mu \mathrm{g} / \mathrm{mL})\end{array}$ & $\mathrm{Cl}_{50}$ \\
\hline FU-NLC & $\mathrm{NA}$ & 6.52 & $\mathrm{NA}$ & $\mathrm{NA}$ \\
C-NLC & $\mathrm{NA}$ & $\mathrm{NA}$ & 0.98 & $\mathrm{NA}$ \\
FU/C-NLC & $20: 1$ & 3.2 & 0.16 & 0.65 \\
FU/C-NLC & $\mathrm{I0:1}$ & 4.9 & 0.49 & 1.25 \\
HA-FU/C-NLC & $20: 1$ & 1.5 & 0.08 & 0.31 \\
HA-FU/C-NLC & $10: 1$ & 3.2 & 0.32 & 0.82 \\
\hline
\end{tabular}

Abbreviations: $\mathrm{IC}_{50}$, half maximal inhibitory concentration; $\mathrm{Cl}_{50}$, combination index calculated when using the $\mathrm{IC}_{50}$ values of the drugs; NLC, nanostructured lipid carriers; FU, fluorouracil; CDDP, cisplatin; C, CDDP-loaded; HA, hyaluronic acid; NA, not applicable.

might be due to the therapeutic effect of NLC formulations (Figure 6). Noticeable body weight losses were observed in free $\operatorname{drug}(\mathrm{s})$ solution formulations and reduction in food intake, energy sag, and inactive movements were observed during the test. In contrast, no significant body weight loss was observed in HA-FU/C-NLC formulation group, demonstrating the reduced systemic toxicity and best tumor inhibition efficiency.

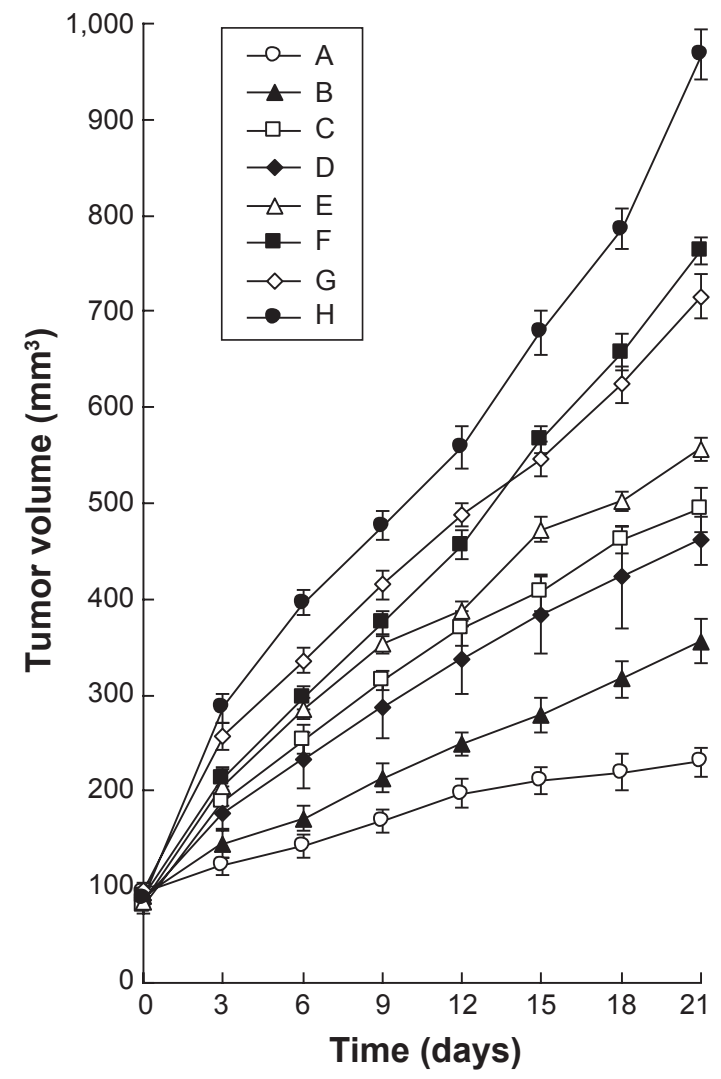

Figure $\mathbf{5}$ In vivo anticancer efficacy presented as tumor volume changes after treatment with different formulations.

Notes: In vivo anticancer efficacy presented as tumor volume changes after treatment with HA-FU/C-NLC (A), FU/C-NLC (B), FU-NLC (C), C-NLC (D), 5-FUstearic acid + CPPD solution (E), 5-FU-stearic acid solution (F), CDDP solution (G), and $0.9 \%$ saline $(\mathbf{H})$ in mice bearing $B G C 823$ human gastric cancer xenograft..

Abbreviations: HA, hyaluronic acid; FU, fluorouracil; C, CDDP-loaded; CDDP, cisplatin; NLC, nanostructured lipid carriers.

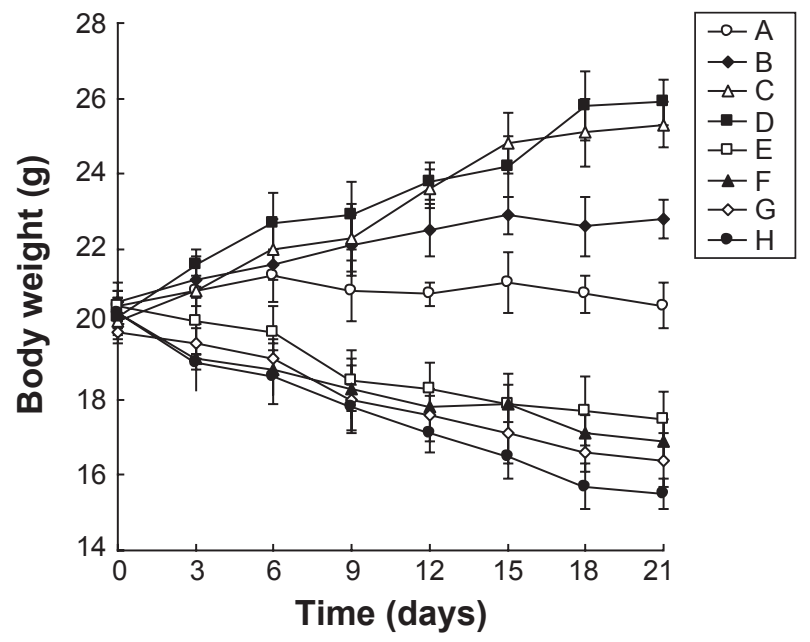

Figure 6 Body weight changes caused by different formulations in BALB/c nude mice bearing BGC823 gastric tumor.

Notes: Body weight changes caused by different formulations in $B A L B / c$ nude mice bearing BGC823 gastric tumor treated with HA-FU/C-NLC (A), FU/C-NLC (B), FU-NLC (C), C-NLC (D), 5-FU-stearic acid + CPPD solution (E), 5-FU-stearic acid solution (F), CDDP solution $(\mathbf{G})$, and $0.9 \%$ saline $(\mathbf{H})$.

Abbreviations: HA, hyaluronic acid; FU, fluorouracil; C, CDDP-loaded; CDDP, cisplatin; NLC, nanostructured lipid carriers.

\section{Conclusion}

In this study, a novel 5-FU-stearic acid prodrug was synthesized and HA-modified NLC were engineered for the effective codelivery of 5-FU and CDDP for the GC combination therapy. The sustained release of drugs from HA-FU/C-NLC with the optimized drug loading ratio enables the synergism in killing GC cells in vitro. The in vivo anticancer experiment showed that HA-FU/C-NLC had the strongest antitumor activity and the least systemic toxicity than free CDDP and uncoated NLC in a gastric tumor bearing animal model. This novel HA-decorated NLC not only showed activity and safety for the delivery of 5-FU and CDDP, but also provided a promising platform for additional formulations of the other two antitumor drugs for cancer therapy.

\section{Acknowledgment}

The work was supported by the Natural Science Foundation of China (NSFC, No 81370485).

\section{Disclosure}

The authors report no conflicts of interest in this work.

\section{References}

1. Jemal A, Bray F, Center MM, Ferlay J, Ward E, Forman D. Global cancer statistics. CA Cancer J Clin. 2011;61(2):69-90.

2. Cappetta A, Lonardi S, Pastorelli D, Bergamo F, Lombardi G, Zagonel V. Advanced gastric cancer (GC) and cancer of the gastro-oesophageal junction (GEJ): focus on targeted therapies. Crit Rev Oncol Hematol. 2012;81(1):38-48.

3. Yang W, Raufi A, Klempner SJ. Targeted therapy for gastric cancer: molecular pathways and ongoing investigations. Biochim Biophys Acta. 2014;1846(1):232-237. 
4. Cunningham D, Allum WH, Stenning SP, et al. Perioperative chemotherapy versus surgery alone for resectable gastroesophageal cancer. N Engl J Med. 2006;355(1):11-20.

5. Shamma RN, Aburahma MH. Follicular delivery of spironolactone via nanostructured lipid carriers for management of alopecia. Int $J$ Nanomedicine. 2014;9:5449-5460.

6. Yang XY, Li YX, Li M, Zhang L, Feng LX, Zhang N. Hyaluronic acidcoated nanostructured lipid carriers for targeting paclitaxel to cancer. Cancer Lett. 2013;334(2):338-345.

7. Luan J, Zhang D, Hao L, et al. Preparation, characterization and pharmacokinetics of Amoitone B-loaded long circulating nanostructured lipid carriers. Colloids Surf B Biointerfaces. 2014;114: 255-260.

8. Mura S, Bui DT, Couvreur P, Nicolas J. Lipid prodrug nanocarriers in cancer therapy. J Control Release. 2015;208:25-41.

9. Negi LM, Talegaonkar S, Jaggi M, et al. Surface engineered nanostructured lipid carriers for targeting MDR tumor: Part I. Synthesis, characterization and in vitro investigation. Colloids Surf B Biointerfaces. 2014;123:600-609.

10. Cho HJ, Yoon HY, Koo H, et al. Self-assembled nanoparticles based on hyaluronic acid-ceramide (HA-CE) and Pluronic ${ }^{\circledR}$ for tumor-targeted delivery of docetaxel. Biomaterials. 2011;32(29):71817190.

11. Han HS, Lee J, Kim HR, et al. Robust PEGylated hyaluronic acid nanoparticles as the carrier of doxorubicin: mineralization and its effect on tumor targetability in vivo. J Control Release. 2013;168(2): 105-114.

12. Ghaffarzadehgan K, Jafarzadeh M, Raziee HR, et al. Expression of cell adhesion molecule CD44 in gastric adenocarcinoma and its prognostic importance. World J Gastroenterol. 2008;14(41):6376-6381.

13. Wang DR, Chen GY, Liu XL, et al. CD44v6 in peripheralblood and bone marrow of patients with gastric cancer as micro-metastasis. World J Gastroenterol. 2006;12(1):36-42.

14. Aghcheli K, Parsian H, Qujeq D, et al. Serum hyaluronic acid and laminin as potential tumor markers for upper gastrointestinal cancers. Eur J Intern Med. 2012;23(1):58-64.

15. Yao HJ, Zhang YG, Sun L, Liu Y. The effect of hyaluronic acid functionalized carbon nanotubes loaded with salinomycin on gastric cancer stem cells. Biomaterials. 2014;35(33):9208-9223.

16. Gomez-Martín C, Lopez-Rios F, Aparicio J, et al. A critical review of HER2-positive gastric cancer evaluation and treatment: from trastuzumab, and beyond. Cancer Lett. 2014;351(1):30-40.

17. Boku N, Yamamoto S, Fukuda H, et al. Fluorouracil versus combination of irinotecan plus cisplatin versus S-1 in metastatic gastric cancer: a randomized phase 3 study. Lancet Oncol. 2009;10(11):1063-1069.

18. Bang YJ, Van Cutsem E, Feyereislova A, et al. Trastuzumab in combination with chemotherapy versus chemotherapy alone for treatment of HER2-positive advanced gastric or gastro-oesophageal junction cancer (ToGA): a phase 3, open-label, randomized controlled trial Lancet. 2010;376(9742):687-697.

19. Cervantes A, Roda D, Tarazona N, Roselló S, Pérez-Fidalgo JA. Current questions for the treatment of advanced gastric cancer. Cancer Treat Rev. 2013;39(1):60-67.

20. Di Paolo A, Danesi R, Falcone A, et al. Relationship between 5-fluorouracil disposition, toxicity and dihydropyrimidine dehydrogenase activity in cancer patients. Ann Oncol. 2001;12(9):1301-1306.

21. Giorgio E, Caroti C, Mattioli F, et al. Severe fluoropyrimidine-related toxicity: clinical implications of DPYD analysis and $\mathrm{UH} 2 / \mathrm{U}$ ratio evaluation. Cancer Chemother Pharmacol. 2011;68(5):1355-1361.

22. Ashwanikumar N, Kumar NA, Nair SA, Kumar GS. 5-Fluorouracillipid conjugate: potential candidate for drug delivery through encapsulation in hydrophobic polyester-based nanoparticles. Acta Biomater. 2014;10(11):4685-4694.

23. van Kuilenburg AB. Dihydropyrimidine dehydrogenase and the efficacy and toxicity of 5-fluorouracil. Eur J Cancer. 2004;40(7): 939-950.

24. Contino C, Maurizis JC, Pucci B. Synthesis and preliminary biological assessments of a new class of amphiphilic telomers bearing 5-fluorouracil moieties. Macromol Chem Phys. 1999;200(6):1351-1355.
25. Nichifor M, Schacht EH, Seymour LW. Polymeric prodrugs of 5-fluorouracil. J Control Release. 1997;48(2-3):165-178.

26. Cui SX, Zhang HL, Xu WF, Qu XJ. 13F-1, a novel 5-fluorouracil prodrug containing an Asn-Gly-Arg $\left(\mathrm{NO}_{2}\right) \mathrm{COOCH}_{3}$ tripeptide, inhibits human colonic carcinoma growth by targeting Aminopeptidase N (APN/ CD13). Eur J Pharmacol. 2014;734:50-59.

27. Thomas AM, Kapanen AI, Hare JI, et al. Development of a liposomal nanoparticle formulation of 5-fluorouracil for parenteral administration: formulation design, pharmacokinetics and efficacy. J Control Release. 2011;150(2):212-219.

28. Ghasemi Z, Dinarvand R, Mottaghitalab F, Esfandyari-Manesh M, Sayari E, Atyabi F. Aptamer decorated hyaluronan/chitosan nanoparticles for targeted delivery of 5-fluorouracil to MUC1 overexpressing adenocarcinomas. Carbohydr Polym. 2015;121:190-198.

29. Li Q, Du YZ, Yuan H, et al. Synthesis of lamivudine stearate and antiviral activity of stearic acid-g-chitosan oligosaccharide polymeric micelles delivery system. Eur J Pharm Sci. 2010;41(3-4): 498-507.

30. Zhu S, Wonganan P, Lansakara-P DS, O’Mary HL, Li Y, Cui Z. The effect of the acid-sensitivity of 4-(N)-stearoyl gemcitabine-loaded micelles on drug resistance caused by RRM1 overexpression. Biomaterials. 2013;34(9):2327-2339.

31. Yu BT, Sun X, Zhang ZR. Enhanced liver targeting by synthesis of N1-stearyl-5-Fu and incorporation into solid lipid nanoparticles. Arch Pharm Res. 2003;26:1096-1101.

32. Rautio J, Kumpulainen H, Heimbach T. Prodrugs: design and clinical applications. Nat Rev Drug Discov. 2008;7(3):255-270.

33. Pabla N, Dong Z. Cisplatin nephrotoxicity: mechanisms and renoprotective strategies. Kidney Int. 2008;73(9):994-1007.

34. Sengupta P, Basu S, Soni S, et al. Cholesterol-tethered platinum IIbased supramolecular nanoparticle increases antitumor efficacy and reduces nephrotoxicity. Proc Natl Acad Sci U S A. 2012;109(28): 11294-11299.

35. Guo S, Miao L, Wang Y, Huang L. Unmodified drug used as a material to construct nanoparticles: delivery of cisplatin for enhanced anti-cancer therapy. J Control Release. 2014;174:137-142.

36. Wang Y, Zhou J, Qiu L, et al. Cisplatin-alginate conjugate liposomes for targeted delivery to EGFR-positive ovarian cancer cells. Biomaterials. 2014;35(14):4297-4309.

37. Liu Z, Rimmer S. Synthesis and release of 5-fluorouracil from poly(Nvinylpyrrolidinone) bearing 5-fluorouracil derivatives. $J$ Control Release. 2002;81(1-2):91-99.

38. Farshori NN, Banday MR, Zahoor Z, Rauf A. DCC/DMAP mediated esterification of hydroxy and non-hydroxy olefinic fatty acids with $\beta$-sitosterol: in vitro antimicrobial activity. Chin Chem Lett. 2010;21(6): 646-650.

39. Fan X, Chen J, Shen Q. Docetaxel-nicotinamide complex-loaded nanostructured lipid carriers for transdermal delivery. Int J Pharm. 2013; 458(2):296-304

40. Luan J, Zhang D, Hao L, et al. Preparation, characterization and pharmacokinetics of Amoitone B-loaded long circulating nanostructured lipid carriers. Colloids Surf B Biointerfaces. 2014;114:255-260.

41. Avgoustakis K, Beletsi A, Panagi Z, Klepetsanis P, Karydas AG, Ithakissios DS. PLGA-mPEG nanoparticles of cisplatin: in vitro nanoparticle degradation, in vitro drug release and in vivo drug residence in blood properties. J Control Release. 2002;79(1-3):123-135.

42. Chou TC. Theoretical basis, experimental design, and computerized simulation of synergism and antagonism in drug combination studies. Pharmacol Rev. 2006;58(3):621-681

43. Yan LH, Wei WY, Cao WL, Zhang XS, Xie YB, Xiao Q. Overexpression of CDX2 in gastric cancer cells promotes the development of multidrug resistance. Am J Cancer Res. 2014;5(1):321-332.

44. Fata F, Ron IG, Kemeny N, O’Reilly E, Klimstra D, Kelsen DP. 5-Fluorouracil-induced small bowel toxicity in patients with colorectal carcinoma. Cancer. 1999;86:1129-1134.

45. Ashwanikumar N, Kumar NA, Nair SA, Kumar GSV. Methacrylicbased nanogels for the $\mathrm{pH}$-sensitive delivery of 5-Fluorouracil in the colon. Int J Nanomedicine. 2012;7:5769-5779. 
46. Liu Z, Rimmer S. Synthesis and release of 5-fluorouracil from poly (N-vinylpyrrolidinone) bearing 5-fluorouracil derivatives. J Control Release. 2002;81:91-99.

47. Platt VM, Szoka FC. Anticancer therapeutics: targeting macromolecules and nanocarriers to hyaluronan or CD44, a hyaluronan receptor. $\mathrm{Mol}$ Pharmacol. 2008;5(4):474-486.

48. Peer D, Margalit R. Loading mitomycin C inside long circulation hyaluronan targeting nano-liposomes increases its antitumor activity in three mice tumor models. Int J Cancer. 2004;108:780-789.

49. Mizrahy S, Raz SR, Hasgaard M, et al. Hyaluronan-coated nanoparticles: the influence of the molecular weight on CD44-hyaluronan interactions and on the immune response. J Control Release. 2011;156: 231-238.
50. Han Y, Zhang Y, Li D, Chen Y, Sun J, Kong F. Transferrin-modified nanostructured lipid carriers as multifunctional nanomedicine for codelivery of DNA and doxorubicin. Int J Nanomedicine. 2014;9: 4107-4116.

51. Harush-Frenkel O, Rozentur E, Benita S, Altschuler Y. Surface charge of nanoparticles determines their endocytic and transcytotic pathway in polarized MDCK cells. Biomacromolecules. 2008;9(2):435-443.

52. Zhao L, Wientjes MG, Au JL. Evaluation of combination chemotherapy: integration of nonlinear regression, curve shift, isobologram, and combination index analyses. Clin Cancer Res. 2004;10(23):7994-8004.

53. Feng T, Tian H, Xu C, et al. Synergistic co-delivery of doxorubicin and paclitaxel by porous PLGA microspheres for pulmonary inhalation treatment. Eur J Pharm Biopharm. 2014;88(3):1086-1093.

\section{Publish your work in this journal}

The International Journal of Nanomedicine is an international, peerreviewed journal focusing on the application of nanotechnology in diagnostics, therapeutics, and drug delivery systems throughout the biomedical field. This journal is indexed on PubMed Central, MedLine, CAS, SciSearch ${ }^{\circledR}$, Current Contents ${ }^{\circledR} /$ Clinical Medicine,
Journal Citation Reports/Science Edition, EMBase, Scopus and the Elsevier Bibliographic databases. The manuscript management system is completely online and includes a very quick and fair peer-review system, which is all easy to use. Visit http://www.dovepress.com/ testimonials.php to read real quotes from published authors. 